\title{
Research on on-orbit SEU characterization of the signal processing platform
}

\author{
Pengcheng Liu ${ }^{a}$, Kefei Xing ${ }^{b}$, Wei He, Zelong Zhang and Wei Deng \\ College of Mechanical Engineering and Automation, National University of Defense Technology, \\ Changsha 410008, China.
}

a18641162300@163.com, b563492379@qq.com

\begin{abstract}
Satellites have suffered many single event upset (SEU) abnormalities in the past decades. To get a detailed SEU characterization of the on-orbit signal processing platform, we installed surveillance programs on 48 signal processing platforms mounted on 24 low-orbiting satellites and monitored the Virtex-II FPGAs. We found 43,213 SEUs at 39,456 monitoring days. The observation window covers a typical rising edge of the 24th solar cycle from March 2010 to April 2015. Results show that the mean SEU rates of $(500 \mathrm{~km}, 700 \mathrm{~km}$ and $1,100 \mathrm{~km})$ orbits are $(0.122,0.318$ and 1.77)/device-day, and the average SEU rate is higher in the solar quiet period. Combined with the proton event data, it is found that a strong solar proton event (SPE) with high proton flux can cause a significant increase in the SEU rate on the $700 \mathrm{~km}$ and $1100 \mathrm{~km}$ orbits. Within one to two days after the maximum peak of the proton flux, the SEU rate reaches the maximum. This finding provides an important reference value for the prediction of the single event upset of signal processing platform. The reliability of the signal processing platform in the space environment can be predicted and evaluated by observing the change of the solar activity for a specific period of time, so as to avoid the space radiation risk by designing the anti - radiation reinforcement scheme.
\end{abstract}

Keywords: Signal processing platform; FPGA; Single event upset; Solar proton event.

\section{Introduction}

The single event upset of electronic devices on satellites has become one of the main factors of the current spacecraft failure, and the sensitivity of the signal processing system on the core of the stars is rising with the improvement of the technology of large scale integrated circuits. FPGA has a large number of internal memory cells (including flip-flop, internal block memory and configuration memory), these storage memory cells will be affected by the impact of high-energy particles generated SEU, thereby affecting the normal operation of the entire system [1]. The solar activity has great influence on space weather, which can lead to the failure of spacecraft. The solar proton events are more active in the solar active period, and the energetic proton flux in the space environment is significantly enhanced, which may be one of the factors leading to the frequent occurrence of single event events [2]. The solar activity is 11-year cycle and is currently in the 24th cycle of solar activity, beginning around the end of 2009. We collected and organized on-orbit SEU data of Virtex-II FPGAs on 48 signal processing platforms mounted on 24 low-orbiting satellites with orbital altitudes of 1100 $\mathrm{km}, 700 \mathrm{~km}$ and $500 \mathrm{~km}$, respectively, and analyzed the SEU characterization. The purpose of this paper is to find out the rule of single event inversion, and then to evaluate the risk of signal processing system from single event effect, and to find a reasonable way to deal with it, which will make a significant contribution to the design of on-orbit signal processing platform.

\section{Data Background}

\subsection{Signal Processing Platform}

All of the 48 signal processing platforms are same except some input/output interfaces. Each satellite has two instruments and flying in the different phases of three different orbits, and each Group of 1- 5 has six instruments distributing in three satellites, and each Group of 6-14 has two in each satellite. Fig. 1 is the instrument diagram and prototype board layout. 


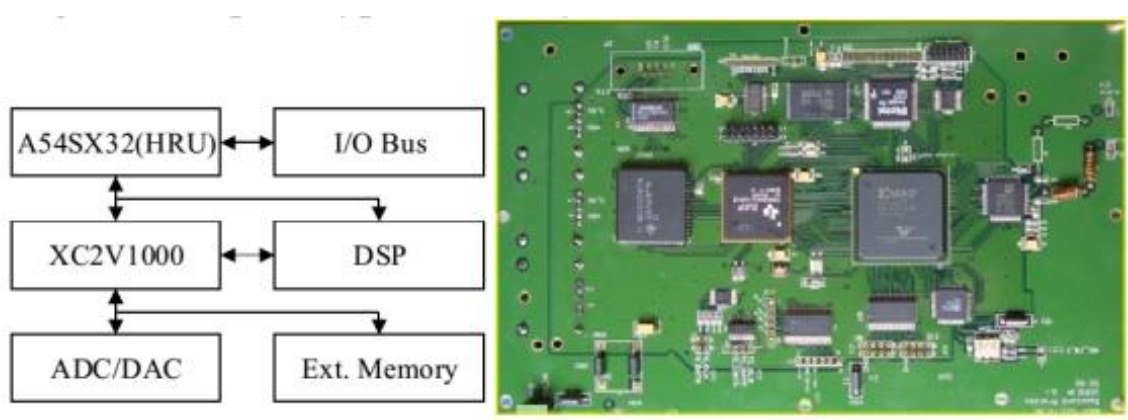

Fig. 1 Diagram of the on-orbit signal processing platform

The instrument contains three main components: an anti-fuse FPGA (A54SX32), which is called High Reliability Unit (HRU) and considered to be less sensitive to SEU; a Virtex-II FPGA (XC2V1000) and a high frequency fixed point DSP (TMS320C6415) ${ }^{[3]}$. All instruments are identical, including assembly position within the satellite, except for some external I/O bus, which has less effect on the SEU data. HRU is used to monitor whether SEU event occurred in Virtex-II FPGA. It reads back and verifies the configuration memory of FPGA at a 30 minutes interval. Every difference will be considered as an SEU event. HRU will record the time and add one to the 4 bits counter, and then scrub to correct the error [3]. Normally the scrub to FPGAs will not abort the current state of them. It is possible to have two or more SEUs in a 30 minutes' verifying cycle, even appearing as multiple-bit upsets (MBU) [4-5]. However, for a long term observation this is unlikely to be a factor, so we assume there is one single upset for each event, and the SEU rate will be underestimated in the worst period.

\subsection{Data Preprocessing}

We defined an observation time coordination whicn based on the Coordinated Universal Time (UTC). The zero point $t_{0}$ is zero hour, zero minute and zero second of March 6th, 2010, which is the time of the first group of observation data. SEUs for each DSP are then accumulated by calendar day. So the observation time $t_{s}$ can be calculated by a $t_{0}$ offset to UTC time $t$. That is to say: $t_{s}=t-t_{0}$.

Supposing $\mathrm{N}_{\mathrm{i}, \mathrm{ts}}$ is the SEU number of instrument $\mathrm{i}$ of all the M Instruments during the observation day of $t_{s}$. Then the mean daily SEU number of $t_{s}$ is

$$
N_{\text {ts }}=\sum_{i=1}^{M} N_{i, t s} / M
$$

In this paper, the minimum unit of time for the day, so the average single device on a single day the average number of SEU is defined as SEU ratet (SEU / device / day).

\section{SEU Characterization}

\subsection{Statistical Distribution for Time}

In order to obtain the intuitionistic time characterization of SEU, the SEU data of 48 signal processing platforms are analyzed statistically. The observed time of each signal processing platform and the number of SEU detected are shown in the following table 1. 
Table 1 Mean SEU Rate of different Signal Processing Platform

\begin{tabular}{|c|c|c|c|c|c|c|c|c|c|}
\hline \multicolumn{10}{|c|}{ Observation } \\
\hline Altitude & Number & Days & SEUs & Rate & Number & Days & SEUs & Rate & $\begin{array}{l}\text { Launch } \\
\text { time }\end{array}$ \\
\hline \multirow{18}{*}{$1100 \mathrm{~km}$} & 01 & 1686 & 194 & 0.115 & 02 & 1687 & 220 & 0.130 & \multirow{3}{*}{ Mar.2010 } \\
\hline & 03 & 1041 & 120 & 0.115 & 04 & 1062 & 136 & 0.128 & \\
\hline & 05 & 1972 & 641 & 0.325 & 06 & 1968 & 542 & 0.275 & \\
\hline & 07 & 1347 & 423 & 0.314 & 08 & 1349 & 478 & 0.354 & \multirow{3}{*}{ Nov.2012 } \\
\hline & 09 & 1359 & 413 & 0.304 & 10 & 1356 & 443 & 0.327 & \\
\hline & 11 & 630 & 182 & 0.289 & 12 & 628 & 229 & 0.365 & \\
\hline & 13 & 372 & 127 & 0.341 & 14 & 372 & 135 & 0.363 & \multirow{3}{*}{ Sep.2013 } \\
\hline & 15 & 191 & 66 & 0.346 & 16 & 187 & 59 & 0.316 & \\
\hline & 17 & 159 & 43 & 0.270 & 18 & 163 & 56 & 0.344 & \\
\hline & 19 & 1858 & 3452 & 1.86 & 20 & 1859 & 3634 & 1.95 & \multirow{3}{*}{ Aug.2014 } \\
\hline & 21 & 1859 & 3260 & 1.75 & 22 & 1859 & 3596 & 1.93 & \\
\hline & 23 & 1859 & 3139 & 1.69 & 24 & 1859 & 3233 & 1.74 & \\
\hline & 25 & 861 & 1310 & 1.52 & 26 & 860 & 1424 & 1.65 & \multirow{3}{*}{ Dec.2014 } \\
\hline & 27 & 861 & 1425 & 1.65 & 28 & 860 & 1542 & 1.79 & \\
\hline & 29 & 860 & 1414 & 1.64 & 30 & 861 & 1480 & 1.72 & \\
\hline & 31 & 583 & 1041 & 1.79 & 32 & 584 & 1049 & 1.80 & Nov.2009 \\
\hline & 33 & 584 & 937 & 1.60 & 34 & 584 & 1103 & 1.89 & July.2011 \\
\hline & 35 & 584 & 1070 & 1.83 & 36 & 584 & 1081 & 1.85 & July.2011 \\
\hline \multirow[t]{4}{*}{$700 \mathrm{~km}$} & 37 & 236 & 399 & 1.69 & 38 & 235 & 413 & 1.76 & July.2013 \\
\hline & 39 & 220 & 347 & 1.58 & 40 & 222 & 387 & 1.74 & Mar.2014 \\
\hline & 41 & 242 & 409 & 1.69 & 42 & 242 & 405 & 1.67 & Sep.2014 \\
\hline & 43 & 117 & 173 & 1.48 & 44 & 118 & 205 & 1.74 & Oct.2014 \\
\hline \multirow{2}{*}{$500 \mathrm{~km}$} & 45 & 119 & 190 & 1.60 & 46 & 119 & 194 & 1.63 & Aug.2010 \\
\hline & 47 & 119 & 170 & 1.43 & 48 & 119 & 224 & 1.88 & May.2012 \\
\hline
\end{tabular}

For the $1,100 \mathrm{~km}$ orbit, there are 38,706 SEUs in 21,927 days, and the mean SEU rate is $1.77 /$ device-day. During the whole period, SEU rate of each device ranges from $1.43 /$ device-day to $1.95 /$ device-day, the variation is $31 \%$. The mean SEU rate of each phase of orbit ranges from 1.63 /device-day to 1.82 /device/day, the variation is $11 \%$. The mean SEU rate in solar quiet period is $1.87 /$ device-day, compared to $1.73 /$ device-day in peak period, the variation is $8 \%$. For the $700 \mathrm{~km}$ orbit, there are 3,837 SEUs in 12,053 days, so the mean SEU rate is 0.318/device-day. During the whole period, mean SEU rate of each device ranges from $0.270 /$ device-day to 0.365 /device-day, the variation is $30 \%$. The mean SEU rate of each phase of orbit ranges from $0.300 /$ device-day to $0.352 /$ device-day, the variation is $16 \%$. The mean SEU rate in solar quiet period is $0.397 /$ device-day, compared to $0.259 /$ device-day in peak period. There is a significant variation of $42 \%$. For the $500 \mathrm{~km}$ orbit, there are 670 SEUs in 5,476 days, and the mean SEU rate is $0.122 /$ device-day. During the whole period, mean SEU rate of each device ranges from 0.115/device-day to 0.130/device-day, the variation is $12 \%$. The mean SEU rate of each phase of orbit is around $0.122 /$ device-day with the variation of $1 \%$. The mean SEU rate in solar quiet period is $0.131 /$ device-day, compared to $0.121 /$ device-day in peak period, the variation is $8 \%$.

The SEU rate of solar calm period and solar active period is calculated respectively in March 2010 as the time division point in the following table 2. 
Table 2 Mean SEU Rate of Solar Quiet Period and Active Period

\begin{tabular}{cccccccc}
\hline \multirow{2}{*}{ Altitude } & & \multicolumn{3}{c}{ Quiet Period } & \multicolumn{3}{c}{ Active Period } \\
& NO. & Days & SEUs & Rate & Days & SEUs & Rate \\
\hline \multirow{5}{*}{$1100 \mathrm{~km}$} & 01 & 309 & 43 & 0.139 & 1377 & 151 & 0.110 \\
& 02 & 309 & 38 & 0.123 & 1378 & 182 & 0.132 \\
& 03 & 595 & 196 & 0.329 & 1377 & 445 & 0.323 \\
& 04 & 595 & 276 & 0.464 & 1373 & 266 & 0.194 \\
& 05 & 481 & 930 & 1.93 & 1377 & 2522 & 1.83 \\
& 06 & 481 & 954 & 1.98 & 1378 & 2680 & 1.94 \\
\hline \multirow{2}{*}{$700 \mathrm{~km}$} & 31 & 481 & 857 & 1.78 & 1378 & 2403 & 1.74 \\
& 32 & 481 & 945 & 1.96 & 1378 & 2651 & 1.92 \\
\hline \multirow{2}{*}{$500 \mathrm{~km}$} & 45 & 481 & 826 & 1.71 & 1378 & 2313 & 1.68 \\
& 46 & 481 & 879 & 1.83 & 1378 & 2354 & 1.71 \\
\hline
\end{tabular}

For $1100 \mathrm{~km}$ orbit, the SEU rate is 0.131 in the solar stationary phase and 0.121 in the solar active phase, and the SEU rate increases by $8 \%$ in the solar stationary phase compared with the active period. For $700 \mathrm{~km}$ orbit, the SEU rate is 0.397 in the solar stationary phase and 0.259 in the solar active phase, and the SEU rate increases by 53\% in the solar stationary phase compared with the active period. For $500 \mathrm{~km}$ orbit, the SEU rate was 1.87 in the solar stationary phase and 1.73 in the solar active phase, and the SEU rate increased by $8 \%$ in the solar stationary phase compared with the active period.

Through the statistical analysis of SEU for solar quite period and active period, the same conclusion is obtained in three different orbits, which the single event is more likely to occur in the solar quite period than in the active period.

\subsection{SEU during Typical Solar Proton Events}

In this paper, four strong solar proton events (from November 2009 to April 2015) are selected as the typical research targets, the details of which are shown in Table 3 [6].

Table 3 Four Typical Solar Proton Events

\begin{tabular}{ccccc}
\hline SPE Number & Start Time & $\begin{array}{c}\text { Duration } \\
\text { (Days) }\end{array}$ & $\begin{array}{c}\text { Max Proton Flux } \\
\text { Start Time }\end{array}$ & $\begin{array}{c}\text { Max Proton Flux } \\
(>10 \mathrm{MeV}) \\
{\left[\mathrm{p} / \mathrm{cm}^{2} \cdot \mathrm{s} \cdot \mathrm{sr}\right]}\end{array}$ \\
\hline 120123 & $2012 / 01 / 2305: 30$ & 6 & $2012 / 01 / 2415: 30$ & 6310 \\
120307 & $2012 / 03 / 0705: 10$ & 8 & $2012 / 03 / 0811: 15$ & 6530 \\
130522 & $2013 / 05 / 2214: 20$ & 8 & $2013 / 05 / 2206: 50$ & 1660 \\
140106 & $2014 / 01 / 069: 15$ & 6 & $2014 / 01 / 0903: 40$ & 1033 \\
\hline
\end{tabular}

The selected 4 typical solar proton events are the strong solar proton events with the maximum flux value of more than 1000pfu in the observed time, and the duration is 6-8 days. Except for 140106, the other solar proton events reached maximum flux peak taken 1-2 days. Because the maximum proton flux of protons in the $10 \mathrm{MeV}$ for 4 typical solar proton events is more than $1000 \mathrm{~cm}^{-2} \cdot \mathrm{s}^{-1} \cdot \mathrm{s}^{-1}$, the energetic particle environment of the earth's radiation belt will be changed. Figure 2-5 compares the change in the SEU rate for each of the four solar proton events.

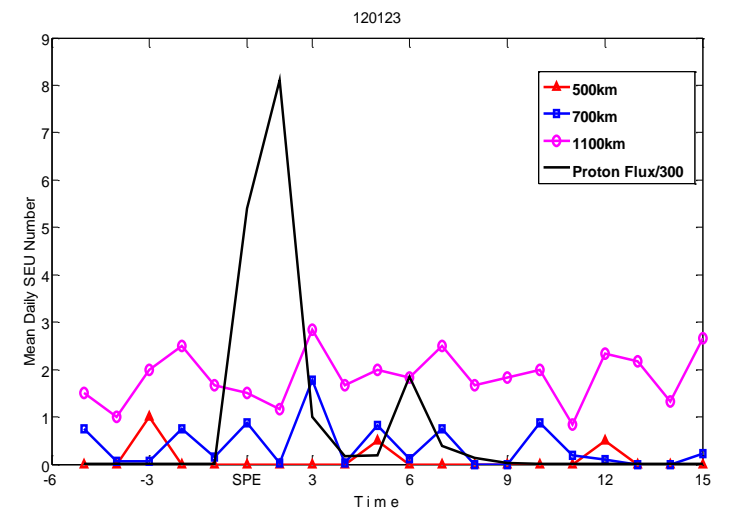

Fig. 2 SEU Rate of NO.12013 SPE

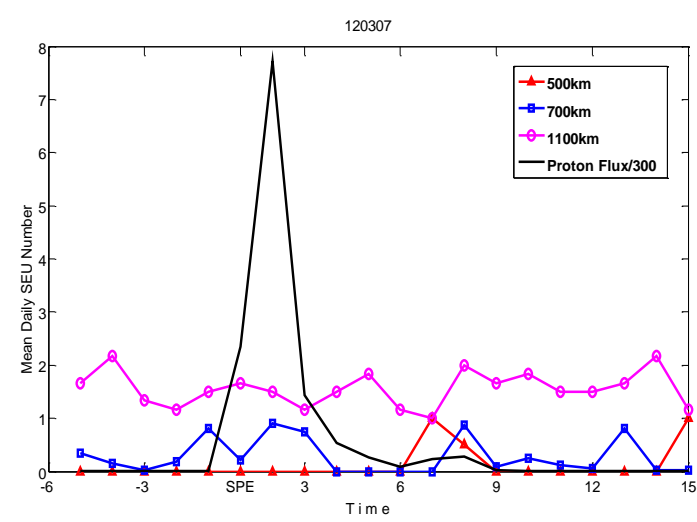

Fig. 3 SEU Rate of NO.12307 SPE 
130522

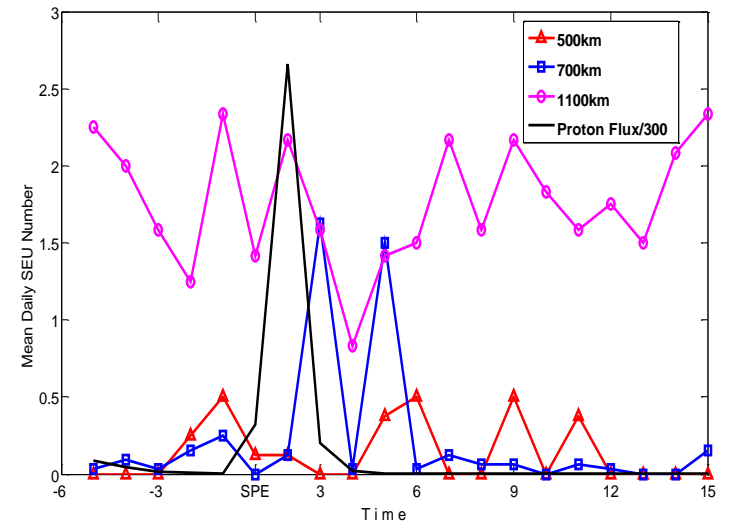

Fig. 4 SEU Rate of NO.13522 SPE

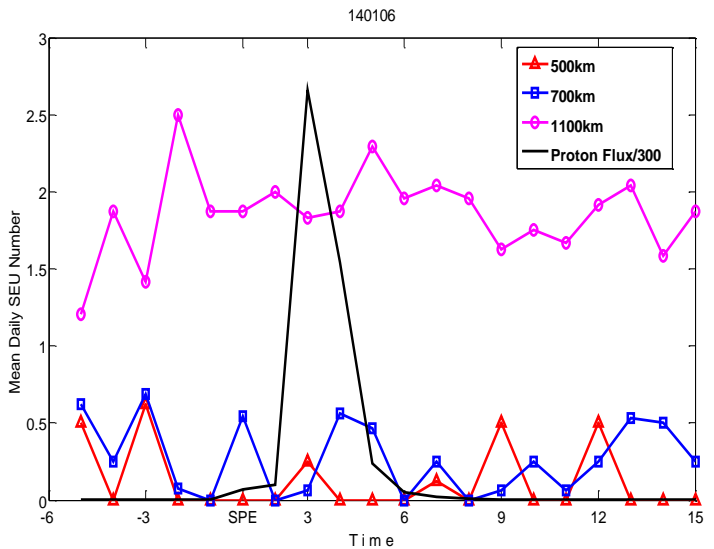

Fig. 5 SEU Rate of NO.140106 SPE

After four typical solar proton events, the SEU rate is obviously improved. The maximum single proton flux of $700 \mathrm{~km}$ and $1100 \mathrm{~km}$ on the day after the maximum proton flux of solar proton event appeared in the 120307 solar proton event. For $700 \mathrm{~km}$ orbit, the maximum proton flux peak of the solar proton event at 120307 was significantly higher than the day before. And the SEU rate of the $1100 \mathrm{~km}$ orbit in the next day has been greatly improved, and the SEU of the $700 \mathrm{~km}$ orbit in the subsequent day has been significantly improved for the 130522 the solar proton event. The SEU rate of $700 \mathrm{~km}$ on the second day after the peak appeared, and the single-particle flip rate on the $1100 \mathrm{~km}$ orbit increased greatly for the 14016 solar proton event.

After these four strong solar proton events, we observed that the SEU rate on the $700 \mathrm{~km}$ and $1100 \mathrm{~km}$ orbit would react instantly and show a significant increase. A better phenomenon is not observed on the $500 \mathrm{~km}$ orbit, which shows the relationship with the solar proton event.

\section{Summary}

This paper analyzes the SEU of 48 on-orbit signal processing platform from different phases on three orbit from 2010 to 2015 . The SEU rate on different orbit is calculated by the statistical analysis method. By analyzed the relationship between the SEU rate and solar activity, the following conclusions are obtained.

There is a negative correlation between the SEU and the solar activity in the Low-Earth orbit, and the SEU is more likely to occur in the solar quite period than in the active period. This indicates that the reliability of SEU effect of on-orbit signal processing platform is more challenged during the solar quite period, and the SEU may be affected by other factors, such as Galactic cosmic ray, which is active during the quiet period. In the follow-up study the correlations between galactic cosmic ray and SEU will be analyzed. This provides a reference for the analysis of factors influencing single event upset.

In the analysis of four typical solar proton events, it is found that the high proton flux of the strong solar proton event can cause a significant increase in the SEU rate on the $700 \mathrm{~km}$ and $1100 \mathrm{~km}$ orbit, and the 1-2 days after the maximum peak of the proton flux, the SEU rate increased rapidly, but had no significant effect on the $500 \mathrm{~km}$ orbit. Therefore, the solar proton event on the satellite SEU has a great threat, we must attach importance to it.

These findings have great reference value for the prediction of the SEU of the on-orbit signal processing platform, the design of anti-radiation reinforcement scheme and the selection of launch time of spacecraft. It is possible to predict the reliability of the signal processing platform in the space environment by predicting the SEU rate of the signal processing platform for a period of time in the future by observing changes in the solar activity. Thus, by designing the reinforcement scheme to be responded (eg. short-time protection, Local standby, etc.) to avoid space radiation risks. It is also possible to select a reasonable launch time for the spacecraft to be launched based on the evaluation results and to reduce the risk due to solar activity disturbances. 


\section{References}

[1] Michael C, Keith M, Diane R, et al. On-orbit flight results from the reconfigurable Cibola Flight Experiment Satellite (CFESat) [C]. IEEE 17th Symposium on Field-Programmable Custom Computing Machines, Napa, USA, April 5-7, 2009.

[2] A. J. Tylka, J. H. Adams, Jr., P. R. Boberg, B. Brownstein, W. F. Dietrich, et al., IEEE Trans. Nucl. Sci. 44, 2150 (1997).

[3] K. Xing, J. Yang, Y. Wang, Z. Xiao, Y. Zhou, Chinese Journal of Astronautics, 28, 123 (2007).

[4] M. Caffrey, K. Morgan, D. Roussel-Dupre, S. Robinson, A. Nelson, A. Salazar, M. Wirthlin, W. Howes, D. Richins, in 17th IEEE Symposium on Field Programmable Custom Computing Machines(FCCM '09), Napa, California, 5-7 April 2009, edited by K. L. Pocek and D. Buell, IEEE Computer Society Press, 2009, pp. 3-10.

[5] H. Quinn, P. Graham, K. Morgan, Z. Baker, M. Caffrey, D. Smith, M. Wirthlin, R. Bell, IEEE Trans. Nucl. Sci. 60, 2682 (2013).

[6] NOAA space environment service center, 2015 http://umbra.nascom.nasa.gov/SEP/seps.html. 